\title{
Performance Evaluation of a Manufacturing Process under Uncertainty using Bayesian Networks
}

\author{
Saideep Nannapaneni ${ }^{1}$, Sankaran Mahadevan ${ }^{* 1}$, Sudarsan Rachuri ${ }^{2}$ \\ ${ }^{1}$ Department of Civil and Environmental Engineering \\ Vanderbilt University, Nashville, TN, USA 37235 \\ ${ }^{2}$ Systems Integration Division, Engineering Laboratory \\ National Institute of Standards and Technology \\ Gaithersburg, MD, USA 20899
}

\begin{abstract}
This paper proposes a systematic framework using Bayesian networks to aggregate the uncertainty from multiple sources for the purpose of uncertainty quantification (UQ) in the prediction of performance of a manufacturing process. Energy consumption, one of the key metrics of manufacturing process sustainability performance, is used to illustrate the proposed methodology. The prediction of energy consumption is not straightforward due to the presence of uncertainty in many process variables and the models used for prediction. The uncertainty is both aleatory (statistical) and epistemic (lack of knowledge); both sources of uncertainty are considered in the proposed UQ methodology. The uncertainty sources occur at different stages of the manufacturing process and do not combine in a straightforward manner, thus a Bayesian network approach is found to be advantageous in uncertainty aggregation. A dimension reduction approach through variance-based global sensitivity analysis is proposed to reduce the number of variables in the system and facilitate scalability in high-dimensional problems. The proposed methodologies for uncertainty quantification and dimension reduction are demonstrated using two examples - an injection molding process and a welding process.
\end{abstract}

\footnotetext{
* Corresponding author. Tel.: +1 6153223040

Email address: sankaran.mahadevan@vanderbilt.edu (S.Mahadevan)
} 
Keywords: Uncertainty quantification; Manufacturing; Bayesian network; Sensitivity analysis;

Energy consumption

Nomenclature

$\begin{array}{cl}\boldsymbol{X} & \text { a vector of random variables } \\ X & \text { a single random variable } \\ \Theta & \text { distribution parameters of a random variable } \\ \theta & \text { a realization of distribution parameters of a random variable } \\ Y & \text { a single output random variable } \\ G & \text { a deterministic function connecting input to output variables } \\ \text { Var () } & \text { Variance operator } \\ E() & \text { Expectation operator } \\ D & \text { Observation data } \\ N_{\text {obs }} & \text { Observed variables in a Bayesian network } \\ \bar{N}_{\text {obs }} & \text { Unobserved variables in a Bayesian network } \\ \text { Pr () } & \text { Probability density function } \\ \text { ABC } & \text { Approximate Bayesian Computation } \\ \text { AHP } & \text { Analytic Hierarchy Process } \\ \text { BN } & \text { Bayesian network } \\ \text { GSA } & \text { Global Sensitivity Analysis } \\ \text { MCMC } & \text { Markov Chain Monte Carlo } \\ \text { UQ } & \text { Uncertainty Quantification }\end{array}$




\section{Introduction}

In recent years, manufacturing processes and production networks have been rapidly pushing the envelope in building complex, optimized products, by taking advantage of new materials, advanced manufacturing techniques, and digital information technology. Along with such developments, it has become increasingly necessary to estimate the economic, social and environmental consequences of the manufacturing activities. Metrics such as energy consumption (in the larger context of sustainability), agility, and asset utilization are being studied to evaluate manufacturing processes and production networks. With the increasing complexity in modern manufacturing processes and production networks, quantification of performance metrics becomes quite complicated. Also, the presence of variability and uncertainty in different processes and their parameters contributes to significant uncertainty in the overall performance prediction. Therefore, it is essential to develop a systematic and rigorous methodology for quantifying the uncertainties in performance prediction in a complex production network. The errors and uncertainty need to be quantified at multiple levels and stages of the manufacturing supply chain, in order to facilitate resource allocation decisions regarding uncertainty reduction and risk management.

Uncertainty sources in various components of the production network may be broadly classified into three categories: natural variability in the manufacturing processes (aleatory uncertainty), information uncertainty due to inadequate, qualitative, missing, or erroneous data (epistemic uncertainty), and modeling uncertainty induced by assumptions and approximations (epistemic uncertainty). The focus of this paper is to develop a systematic methodology that quantifies the overall uncertainty in the computation of performance metrics as well as the 
contributions of individual sources of uncertainty to the overall uncertainty in the metrics assessment.

Quantification of uncertainty in manufacturing performance prediction in the manufacturing domain has been previously attempted using fuzzy set theory (Reza et al; 2013) and Monte Carlo simulations (Pehlken et al; 2015, Sonnemann et.al; 2003). This paper pursues the Bayesian network approach for this problem. The Bayesian network allows the integration of various types of uncertainty that (a) occur at different stages of the life cycle, and (b) combine in different ways (linear, nonlinear, coupled, nested, and iterative) (Sankararaman et al; 2011b, Liang and Mahadevan; 2011). Information about various uncertainty contributors is available in heterogeneous formats and fidelity, from multiple sources (e.g., test data, expert opinion, operational data, legacy system data, and model-based simulations). The Bayesian network offers a systematic and rigorous approach for uncertainty integration and management, making use of all available heterogeneous information (Bartram and Mahadevan; 2014). The term "uncertainty integration" here implies the aggregation of uncertainty resulting from multiple sources.

Numerous applications of statistical process control have dealt with variability and quality control in manufacturing, by monitoring the variance of product features or manufacturing process parameters. The advantage with a Bayesian network (BN) approach is that it allows for multiple analyses such as sensitivity analysis, dimension reduction, resource allocation, model calibration, and forward uncertainty propagation, by fusing heterogeneous information. The methodology in this paper seeks to exploit these features of the $\mathrm{BN}$ for manufacturing applications. 
Probabilistic graphical models such as Bayesian networks have shown much effectiveness in the aggregation of uncertainty information across complex networks in many application domains, such as information retrieval, data fusion and engineering decision-making (Dahll; 2000), safety assessment of software-based systems (De Campos et al; 2004), computational biology and bioinformatics (Friedman et al; 2004, Jiang et al; 2011), epidemiology (Xiang and Cooper; 2010), and civil infrastructure networks (Bensi and Der Kiureghian; 2010). Discrete probabilities have been considered in many applications of $\mathrm{BN}$ to risk analysis and decisionmaking (Castillo et al; 1999, Mahadevan et al; 2001), whereas recent work is expanding the BN approach to uncertainty quantification, diagnosis and prognosis using both discrete and continuous probabilities. The use of a BN for uncertainty aggregation across multiple physics and scales, and using experimental data at multiple levels, has been illustrated for mechanical systems (Urbina et al; 2012) and microelectromechanical systems (MEMS) devices (Ling and Mahadevan; 2013). Recent work has also extended the use of BN towards resource allocation decision making, in selecting the optimum combination of tests needed to meet the target uncertainty level in model prediction (Sankararaman; 2013b).

The above research has focused mostly on the analysis of physical systems, not manufacturing environments involving multiple processes. So far, Bayesian networks have been used in the manufacturing domain for fault diagnosis (McNaught and Chan; 2011, Rodrigues et al; 2000) and discrete-event reliability modeling (Weber and Jouffe; 2006), but the focus in this paper is on information fusion, calibration of uncertain parameters, uncertainty reduction and handling of both discrete and continuous variables for performance prediction. Note that fault diagnostics and monitoring are focused on measurement and inference about the current state. Prediction involves forward uncertainty propagation, which has to include the contributions of both aleatory 
and epistemic variables. The BN framework accommodates both measurement and prediction as discussed in Section 2.

The Bayesian network enables analyses in two directions: (1) forward and (2) inverse. Forward propagation through the Bayesian network aggregates information from all available sources (e.g., models, data, expert opinion) to quantify the uncertainty in the overall performance metric of the manufacturing process, such as cost, material or energy consumption, $\mathrm{CO}_{2}$ emission etc. Several types of inverse problem are of interest: data analytics, model calibration, fault diagnosis, process and quality control, resource allocation, and performance optimization; all these are enabled by the Bayesian network (Sankararaman; 2012).

Along with Bayesian network construction, variance-based global sensitivity analysis (explained in Section 2.C) can be carried out to identify the dominant variables affecting the uncertainty in the output; the variables (either input variables or model parameters) with low sensitivity indices can be assumed to be deterministic at their nominal or most probable values. In addition, the contributions of aleatory and epistemic uncertainty sources can be individually distinguished, using global sensitivity analysis. Sankararaman and Mahadevan (2013a) assessed the contributions of aleatory and epistemic sources of uncertainty within a single random variable and illustrated the method with two simple mathematical examples. In this paper, that approach is extended for networks with multiple models and variables (some aleatory, some epistemic and some variables with both aleatory and epistemic uncertainty). Distinguishing the aleatory and epistemic contributions guides resource allocation towards uncertainty reduction, since only epistemic uncertainty is reducible.

The main contributions of this paper can be summarized as follows - (1) Development of an uncertainty quantification framework using Bayesian networks for the performance evaluation of 
manufacturing processes; (2) Development of a dimension reduction technique using variancebased global sensitivity analysis, clearly distinguishing the contributions of aleatory and epistemic uncertainty sources; and (3) Illustration of the developed techniques for two manufacturing processes - injection molding and welding.

The remainder of this paper is organized as follows. Section 2 provides an introduction to epistemic sources of uncertainty, Bayesian networks and sensitivity analysis. Section 3 describes the methodology for characterizing the uncertainty in manufacturing performance metrics using Bayesian networks, and Section 4 discusses dimension reduction using sensitivity analysis. In Section 5, two examples are provided to demonstrate the proposed methodologies in energy consumption in welding and injection molding. Concluding remarks are provided in Section 6.

\section{Background}

This section provides brief introductions to epistemic uncertainty representation due to data and modeling sources, Bayesian networks and variance-based sensitivity analysis. These techniques are later used in the uncertainty quantification methodology developed in Section 3.

\subsection{Representation of epistemic uncertainty}

Here, procedures for the representation and quantification of epistemic uncertainty due to data (data uncertainty) and model (model uncertainty) are discussed in order to facilitate UQ using a Bayesian network.

\subsubsection{Data Uncertainty}

One of the challenges in manufacturing process assessment is in collecting and processing information about the quantities to be used in the calculation of performance metrics. Such information could be sparse, imprecise, qualitative, subjective, faulty, or missing leading to 
uncertainty in the distribution parameters and distribution type of the variable being studied. In the context of a probabilistic framework, the Bayesian approach is naturally suited to handle such data cases and update the description with new information. For example, consider a material property, like Young's modulus, being represented using a probability distribution with some parameters. In the presence of sufficient data, the distribution parameters can be inferred precisely but when data are insufficient, the distribution parameters are uncertain, and this epistemic uncertainty is represented using probability distributions in the Bayesian approach, and updated as additional information becomes available. Flexible parametric or non-parametric probability distributions can be used within the Bayesian framework to represent such epistemic uncertainty. Several four-parameter distributions (such as beta, gamma, Johnson and Pearson) have flexible shapes and can be used to fit a variety of datasets by modifying the values of the parameters. Either maximum likelihood or Bayesian estimation can be used to construct the parametric or non-parametric distribution. (Sankararaman and Mahadevan; 2011a).

\subsubsection{Model Uncertainty}

Model uncertainty refers to the uncertainty in the computational models that are used to connect input and output variables in the manufacturing process. The models could be based on first principles (physics-based models) or based on data (data-driven models). Model uncertainty arises from three types of sources namely, model parameters, model form, and solution approximations. The corresponding procedures to quantify them are calibration, validation and verification. Model parameters are estimated using calibration data, and Bayesian calibration constructs posterior probability distributions for the model parameters. Model form uncertainty may be quantified in two ways: either through a validation metric, based on validation data, or as model form error (also referred to as model discrepancy or model inadequacy) during calibration. 
Model form error can be estimated along with the model parameters using calibration and/or validation data, based on the comparison of model prediction against physical observation, after accounting for solution approximation errors, uncertainty quantification errors, and measurement errors in the inputs and outputs (Rebba et al; 2006, Sankararaman and Mahadevan; 2012).

Solution approximation errors include the errors in solving the computational model itself such as discretization error and surrogate model error (Liang and Mahadevan; 2011). Discretization error arises when the solution of a continuum domain is computed using numerical techniques (e.g., finite element methods) which involve discretization of the continuum domain. Discretization error can be quantified by comparing solutions with different levels of discretization (Richards; 1997, Rangavajhala et al; 2011). Surrogate models are often used when high fidelity physics models are computationally expensive. The estimation of surrogate model error involves comparing the output of the original computational model with the surrogate model. Note that the original model already needs to be run multiple times to generate training points to construct the surrogate model; the surrogate model error estimation requires a few additional runs of the original model.

\subsection{Bayesian Networks}

A Bayesian network is a probabilistic graphical model, consisting of nodes and arcs, where nodes represent the variables in the system and arcs represent the conditional probability distributions between nodes. Mathematically, a Bayesian network is the joint probability distribution of a set of variables $\boldsymbol{X}=\left(X_{1}, X_{2} \ldots X_{n}\right)$ represented as

$$
\operatorname{Pr}_{B}(\boldsymbol{X})=\prod_{i=1}^{n} \operatorname{Pr}_{B}\left(X_{i} \mid \Pi_{X_{i}}\right)
$$


where $\Pi_{X_{i}}$ represents the set of parent nodes of $X_{i}$ and $\operatorname{Pr}_{B}\left(X_{i} \mid \Pi_{X_{i}}\right)$ represents the conditional probability distribution of $X_{i}$, given its parent nodes. If $X_{i}$ has no parent nodes, then $P r_{B}\left(X_{i} \mid \Pi_{X_{i}}\right)$ represents the marginal probability distribution of $X_{i}$.

For illustration, consider the Bayesian network shown in Fig. 1, which represents the joint probability distribution of three random variables A, B, C. The joint probability distribution can be represented as (using Eq. 1)

$$
\operatorname{Pr}_{B}(A, B, C)=\operatorname{Pr}(A) \operatorname{Pr}(B) \operatorname{Pr}(C \mid A, B)
$$

Since $A, B$ have no parent nodes, marginal probability distributions are used for them instead of conditional probability distributions. As stated in Section 1, a BN enables two types of analyses - (1) forward, and (2) inverse. For this example, the forward analysis involves computation of uncertainty in $C$ given the uncertainty in root nodes (nodes with no parent nodes) $-A$ and $B$. Among several inverse problems using Bayesian networks, model calibration is quite common and considered in this paper. For this example, model calibration involves updating the marginal distributions of $A$ and $B$ when any new data are available on $C$.

Consider the case when only data are available on variable $B$, rather than the marginal probability distribution. In the case when only data are available on variable $B$, either parametric or non-parametric probability distributions can be fit to the available data (see Section 2.1.1). Using the fit probability distribution, further analysis, either forward or inverse, can be carried out.

The conditional probability distribution associated with $C(\operatorname{Pr}(C \mid A, B))$ can be interpreted as a stochastic model connecting variables $A, B$ with $\mathrm{C}$. Presence of model errors results in the uncertainty of the conditional probability distributions. Analyses such as model verification, model validation can be performed to quantify the model errors and the revised model after 
accounting for all the model errors can be used as the conditional probability distribution in the Bayesian network. Thus, the results of data and model uncertainty can be integrated into the Bayesian network.

\subsection{Sensitivity Analysis}

Sensitivity analysis is carried out to estimate the contribution of each source of uncertainty to the overall uncertainty in the model prediction. Two types of sensitivity analysis are available local and global (Liang and Mahadevan; 2011). The local sensitivity index of a variable (input variable, model parameter or model error term) measures the variation of the model prediction when the variable is fixed at a single value whereas the global sensitivity index measures the variation of model prediction when the variable is varied over its probability distribution. Global sensitivity analysis is used in this paper as it considers the entire probability distribution of a variable in assessing the uncertainty contribution to the output.

Consider a model with $n$ random input variables $X_{1}, X_{2}, \ldots, X_{n}$ given by

$$
Y=G\left(X_{1}, X_{2}, X_{3}, \ldots, X_{n}\right)
$$

The method of global sensitivity analysis is based on the variance decomposition theorem, where the variance in an output variable is decomposed as

$$
\operatorname{Var}(Y)=E_{X_{i}}\left(\operatorname{Var}_{X_{\sim i}}\left(Y \mid X_{i}\right)\right)+\operatorname{Var}_{X_{i}}\left(E_{X_{\sim i}}\left(Y \mid X_{i}\right)\right)
$$

where $Y$ represents the output variable (model prediction), $X_{i}$ is the variable for which the sensitivity measures are computed, and $X_{\sim i}$ represents all the other variables excluding $X_{i}$. Two indices are computed for each variable - main effect, also called the first-order effect, and total effect. The first-order effect index $\left(S_{i}^{I}\right)$ quantifies the individual contribution from the 
uncertainty in an input variable $X_{i}$ (without considering its interaction with other variables), to the overall uncertainty in the output variable and is given by

$$
S_{i}^{I}=\frac{\operatorname{Var}_{X_{i}}\left(E_{X_{\sim i}}\left(Y \mid X_{i}\right)\right)}{\operatorname{Var}(Y)}
$$

where $E_{X_{\sim i}}\left(Y \mid X_{i}\right)$ calculates the expected value of the model output $Y$ when $X_{i}$ is fixed at a specific value, and $\operatorname{Var}_{X_{i}}$ computes the variance of this expected value when the randomness in $X_{i}$ is included. The contribution from the variable $X_{i}$ including its interaction with all other variables is quantified by the total effects index, computed as

$$
S_{i}^{T}=1-\frac{E_{X_{\sim i}}\left(\operatorname{Var}_{X_{i}}\left(Y \mid X_{\sim i}\right)\right)}{\operatorname{Var}(Y)}
$$

where $\operatorname{Var}_{X_{i}}\left(Y \mid X_{\sim i}\right)$ computes the variance of $Y$ when all the input variables except for $X_{i}$ are fixed at specific values, and $E_{X_{\sim i}}$ calculates the expected value of this variance considering the randomness in $X_{\sim i}$. Note that superscripts $I$ and $T$ on $S_{i}$ in Eq. (5) and (6) are used to represent individual effects and total effects respectively. The superscript $T$ should not be confused with the transpose operation used in matrix algebra.

\section{Uncertainty Quantification}

The procedure for uncertainty quantification can be divided into the following steps:
A. Construction of the Bayesian network
B. Bayesian inference (inverse analysis)
C. Forward uncertainty propagation (forward analysis)

The steps are described below. 


\subsection{Construction of the Bayesian network}

There are three ways to construct a Bayesian network - a) from physics-based knowledge about the system; b) from data by using learning algorithms (Heckerman et al; 1995); and c) a hybrid approach using both physics-based knowledge and learning algorithms. In the hybrid approach, the physics knowledge is used to capture some of the dependencies between the variables and available data are used to capture the remaining dependencies. This approach is useful when building a Bayesian network for a collection of manufacturing processes in a production network. Physics-based models might be available for individual processes but when individual processes are composed to form a production network, some of the interactions may be unknown (For example, consider two simple systems - a heating system and a pipe to transport a flammable liquid. The two systems when separated by a large distance may function properly but when the heating system is brought close to the pipe, the pipe gets heated, thereby heating up the flammable liquid and eventually causing fire).

The task of learning a BN consists of two steps - (1) Structure learning: Finding a graphical structure that represents the dependence between nodes based on available data (i.e., identify the parent and child nodes); and (2) Parameter learning: Quantify the dependence between the nodes by estimating the conditional probability distributions. Structure learning algorithms can be broadly divided into three categories (Scutari; 2010) - (1) constraint-based, also called conditional independence methods, (2) score-based, and (3) hybrid. The constraint-based algorithms use conditional independence tests to learn the structure of the BN. In score-based methods, each possible BN structure is assigned a score based on the goodness of fitting available data and heuristic optimization (e.g., hill-climbing) is used to obtain the structure that maximizes the score. Hybrid algorithms employ both conditional independence tests and 
network scores in learning the structure of the BN. The conditional independence tests are used to reduce the space of possible BN structures whereas score-based methods are used to obtain the optimal structure among them. Once the structure of the Bayesian network is determined, the next task is parameter learning. The parameters of conditional probability distributions can be estimated from available data using the maximum likelihood approach (Scutari; 2010).

\subsection{Bayesian inference}

Following the $\mathrm{BN}$ construction is the Bayesian inference procedure. The important idea in this step is to estimate the posterior distributions of the unknown parameters using observation data that may be available on several variables in the $\mathrm{BN}$. Let $D$ represent the observation data available for a subset $\left(\boldsymbol{N}_{\text {obs }}\right)$ of the nodes in BN. The joint probability distribution of the other nodes $\left(\overline{\boldsymbol{N}}_{\boldsymbol{o b s}}\right)$ in the BN can be updated based on Bayes' theorem as

$$
\operatorname{Pr}\left(\overline{\boldsymbol{N}}_{\boldsymbol{o b s}} \mid \boldsymbol{N}_{\boldsymbol{o b s}}=D\right)=\frac{\operatorname{Pr}\left(\boldsymbol{N}_{\boldsymbol{o b s}}=D \mid \overline{\boldsymbol{N}}_{\boldsymbol{o b s}}\right) \operatorname{Pr}\left(\overline{\boldsymbol{N}}_{\boldsymbol{o b s}}\right)}{\int \operatorname{Pr}\left(\overline{\boldsymbol{N}}_{\boldsymbol{o b s}}, \boldsymbol{N}_{\boldsymbol{o b s}}=D\right) d \overline{\boldsymbol{N}}_{\boldsymbol{o b s}}}
$$

In the above equation., $\operatorname{Pr}\left(\overline{\boldsymbol{N}}_{\boldsymbol{o b s}}\right)$ and $\operatorname{Pr}\left(\overline{\boldsymbol{N}}_{\boldsymbol{o b s}} \mid \boldsymbol{N}_{\boldsymbol{o b s}}=D\right)$ are the prior and posterior probability distributions of $\overline{\boldsymbol{N}}_{\boldsymbol{o b s} \boldsymbol{s}} \cdot \operatorname{Pr}\left(\boldsymbol{N}_{\boldsymbol{o b s}}=D \mid \overline{\boldsymbol{N}}_{\boldsymbol{o b s}}\right)$ is the likelihood function and the denominator is a normalizing constant. In most cases, it is intractable to obtain an analytical expression for the posterior distribution and therefore, numerical techniques such as Markov Chain Monte Carlo (MCMC) sampling (Brooks; 1998) and Approximate Bayesian Computation (ABC) (Toni et al; 2009) are used to generate samples from the posterior distribution. MCMC method (Metropolis-Hastings algorithm) is used in this paper.

\subsection{Forward uncertainty propagation}

The next step after calibration of model parameters is forward uncertainty propagation to obtain the distribution of the output quantity of interest (e.g., energy consumption in a manufacturing 
process). Samples of the input variables and model parameters are generated from their distributions and propagated through the network using Monte Carlo sampling and corresponding samples of the output are obtained. These samples are used to construct the probability distribution of the output quantity of interest. (Note that posterior distributions are used for those nodes updated by the previous step of Bayesian inference). Fig. 2 describes the above uncertainty quantification procedure.

\section{Sensitivity Analysis for Dimension and Uncertainty Reduction}

Sensitivity analysis can be performed to identify the dominant variables contributing to the uncertainty in the output variables. Also, separating the contributions of aleatory and epistemic uncertainty sources to the output quantity of interest can help to determine if the uncertainty of the output could be reduced by allocating more resources for data collection or model improvement, by identifying the dominant epistemic uncertainty sources, since only epistemic uncertainty is reducible. Thus, sensitivity analysis helps to answer two questions: (1) Which variables have a dominant influence on the output uncertainty? (2) On which variables should resources for data collection be directed to reduce the output uncertainty?

\subsection{Dimension reduction}

The dimension of the Bayesian network (i.e., number of nodes and the edges connecting them) for a production network increases with the number of individual manufacturing processes. With increased number of processes the number of parameters to be calibrated might also increase. The computational effort increases with the number of variables in the network. Therefore it is desirable to have a reduced set of variables without compromising the accuracy in output statistics estimation. 
Dimension reduction techniques in the literature are classified into two types - filter approach (Saeys et al; 2007) and wrapper approach (Kohavi and John; 1997). In the filter approach, all input variables are ranked according to a ranking criterion and the most significant variables can be selected. The number of variables selected depends on the analysis requirements and accuracy. In the wrapper method, a subset of variables is selected, from all possible subsets of variables that best describe the output quantity of interest. An optimization search technique is generally used to obtain the best subset of variables. The proposed dimension reduction technique is based on variance-based global sensitivity analysis and falls under the filter approach.

During the inference stage, the prior distributions of all the variables in the system are available and GSA can be performed using the prior distributions of input variable to the output quantity of interest. Here, the ranking criterion corresponds to the sensitivity indices using the prior distributions. A threshold value of sensitivity index can be assumed and all the variables whose sensitivity indices are less than the threshold value can be considered deterministic. The threshold can be decided based on the relative amount of output variance contributed by different variables and the available computational resources. The equations for computing the sensitivity indices were provided in Section 2. The deterministic value of the insensitive variables can be nominal values or the most probable value from their corresponding prior distributions. Using the reduced set of variables, calibration can be performed and posterior distributions of the variables can be obtained. Using the posterior distributions, GSA can be carried out to further reduce the number of variables that have significant influence on the output variable. Fig. 3 describes the dimension reduction methodology using sensitivity analysis.

\subsection{Uncertainty reduction}


In some cases, the parameters in the probability distributions used to represent the variables can also be uncertain and are themselves represented using probability distributions; thus the same variable has both aleatory and epistemic uncertainty, where the epistemic uncertainty is due to the uncertain distribution parameters. It is often desirable to separate the variance contributions of aleatory and epistemic components in order to determine which uncertainty component (aleatory or epistemic) is the dominant contributor to the output variance. Since aleatory uncertainty is irreducible and epistemic uncertainty is reducible, data collection resources can be allocated to variables with dominant epistemic contribution. To separate the contributions of the aleatory and epistemic components of uncertainty in a random variable $X$ with uncertain distribution parameters $\Theta$, Sankararaman and Mahadevan (2013a) proposed the use of an auxiliary variable $u$, based on the probability integral transform, to represent the aleatory component as

$$
u=F_{X}(x \mid \Theta=\theta)=\int_{-\infty}^{x} f_{X}(w \mid \Theta=\theta) d w
$$

where $\theta$ is a realization of the distribution parameter $\Theta, w$ is a dummy variable used for integration, and $F_{X}(x \mid \Theta=\theta)$ denotes the CDF value of variable $X$ for a realization of $\Theta$. The variability in $X$ is represented by this auxiliary variable $u$, which follows a uniform distribution between 0 and 1 (since the CDF values range from 0 to 1 ). Global sensitivity analysis requires a deterministic functional relationship between the input random variables and the output. In the presence of both aleatory and epistemic uncertainty, the variable $X$ has to be represented by a family of distributions. Each realization of the distribution parameters results in a probability distribution of $X$. Using a realization of $\mathrm{u}$, one can obtain a unique value of $X$. Thus the use of the auxiliary variable results in a deterministic functional relationship between $(u, \Theta)$ and the 
variable $X$, and thus GSA can be carried out to quantify the contribution of $u$ (aleatory) and $\Theta$ (epistemic) to the variance of $X$.

The above idea can be extended further to sensitivity analysis of the model output also. In the presence of model uncertainty, a specific realization of the input $X$ results in a probability distribution of the output $Y$. An additional auxiliary variable $u_{m}$ can be defined to develop a deterministic relationship between $\left(X, u_{m}\right)$ and $Y$. Now, GSA can be carried out to quantify the contribution of $X$ and $u_{m}$ to the variance of $Y$.

This paper extends this idea to a network of multiple models and variables, some aleatory, some epistemic, and some with both aleatory and epistemic uncertainty components. In a realistic problem, there is no simple way to combine the contributions of these different uncertainty sources. The Bayesian network helps to systematically to track the contributions of various uncertainty sources and perform the sensitivity analysis.

The key novel elements of this paper's use of BN for UQ in manufacturing are as follows:

(1) The ability of $\mathrm{BN}$ to fuse information from mathematical models and available data are exploited to facilitate the calibration of uncertain model parameters.

(2) The $\mathrm{BN}$ is developed in a manner that can handle both discrete and continuous variables. The continuous variables are allowed to have any arbitrary distributions, instead of Gaussian.

(3) GSA is used for dimension reduction, in order to address the challenge of scalability in highdimensional problems.

(4) GSA is extended to inform resource allocation for uncertainty reduction, by separating the contributions of aleatory and epistemic sources to the overall performance uncertainty. 


\section{Examples}

In this section, the proposed uncertainty evaluation methodology is demonstrated using two manufacturing processes - welding and injection molding. Welding can be considered as a single process whereas injection molding can be considered as a composition of three sub-processes (melting the polymer, injecting the polymer into the mold and cooling the polymer). Thus the $\mathrm{BN}$ for injection molding can be composed by combining the BNs of the sub-processes; thus injection molding is used to provide an illustration of $\mathrm{BN}$ composition. This idea could be extended to manufacturing networks consisting of multiple processes. As shown later, aleatory uncertainty is dominant in welding whereas epistemic uncertainty is dominant in injection molding. Thus the uncertainty reduction resource allocation decisions are different in the two examples.

\subsection{Welding process}

This example illustrates uncertainty quantification in the evaluation of the theoretical minimum energy consumption of a welding process (Feng et al; 2014). From Fig. 4, the volume of a weld is given by the following equation.

$$
V=L \times\left(\frac{3}{4} \times l h+g t+\frac{(l-g)}{2} \times(t-e)\right)
$$

where $\mathrm{V}$ and $\mathrm{L}$ represent the volume of the weld and the length of the weld respectively. All the other parameters are labeled in Fig. 4 (Feng et al; 2014).

For the sake of illustration, the filler and the metal are assumed to be same material. The total minimum theoretical energy required for the process is given as

$$
E_{A P}^{T R}=\rho\left(C_{p}\left(T_{f}-T_{i}\right)+H\right) V
$$


where $E_{A P}^{T R}, \rho, C_{p}, T_{f}, T_{i}, H$ represent the theoretical minimum energy, density of the material, heat capacity, final and initial temperatures, and latent heat of the material. $\mathrm{A} B N$ is constructed to represent the causal dependencies between the variables in the network (in Eqs. 9, 10), and is shown in Fig. 5. The variables with aleatory uncertainty and known distribution parameters are: length of the weld $(L)$, density $(\rho)$, latent heat $(H)$, heat capacity $\left(C_{p}\right)$, initial and final temperatures $\left(T_{i}, T_{f}\right)$, and weld parameters $(g, e)$. In addition, there are some parameters $(l, h, e)$ with aleatory uncertainty but unknown distribution parameters (means $\mu_{l}, \mu_{h}, \mu_{e}$ and standard deviations $\left.\sigma_{l}, \sigma_{h}, \sigma_{e}\right)$. These unknown distribution parameters are calibrated using observation data.

In the above Bayesian network, data are assumed to be available on the weld parameters $(l, h, e)$. The quantities to be calibrated include the distribution parameters of weld parameters $(l, h, e)$. A synthetic dataset of 100 observations is created for calibration using available mathematical models for welding along with some measurement error and the statistics of variables used for the generation of the dataset are provided in Table 1.

The error in the measurement of weld parameters $(l, h, e)$ is assumed to have a normal distribution with zero mean and a standard deviation of $0.01 \mathrm{~mm}$. The assumed prior distributions for the calibration parameters are provided in Table 2. Uniform priors are assumed, since only lower and upper bounds are available.

MCMC sampling (Metropolis-Hastings algorithm) is used to generate samples from the posterior distribution of the parameters. The samples from the posterior distributions are then used to estimate the probability distribution of theoretical energy consumption $\left(E_{A B}^{T R}\right)$ using Monte Carlo sampling and kernel density estimation as described in Section 3. The plots 
showing the prior and posterior distributions of the calibration parameters and theoretical minimum energy are shown in Fig. 6.

GSA is carried out using both the prior and posterior distributions to demonstrate the proposed dimension reduction method, and results are shown in Table 3. As stated earlier, variables $l, h, e$ have both aleatory and epistemic uncertainty (uncertain distribution parameters). The epistemic uncertainty is represented using assumed prior probability distributions, as shown in Table 2. The auxiliary variable for variable $l$ is represented by $u_{l}$ and similar notation is used to represent the auxiliary variables of $h$ and $e$. From the results of sensitivity analysis using prior distributions, it can be observed that the epistemic quantities - standard deviations of weld parameters $(l, h, e)$, have very low first-order sensitivity $(<0.001)$, i.e., very low contribution to the energy consumption variance. These variables could therefore be assumed to be deterministic at their most probable value as stated in Section IV. But the variables are still included in model calibration to show that their sensitivity values further reduce upon model calibration (see Table 3). Therefore, assuming such variables as deterministic saves computational resources without compromising the statistics of the output quantity of interest, and thus significantly facilitates sensitivity.

\subsection{Injection Molding}

This example illustrates uncertainty quantification in the energy consumption for an injection molding process. The injection molding process (Madan et al; 2013) can be divided into three stages - a) melting of polymer; b) injection of polymer into the mold; c) cooling of polymer to form product.

\subsubsection{Melting process}


In this process, the polymer which initially is in solid state is heated beyond the melting point to turn it into liquid state. The power used in melting the polymer $\left(P_{\text {melt }}\right)$ is given by (Madan et $\mathrm{al} ; 2013)$

$$
P_{\text {melt }}=\rho \times Q_{\text {avg }} \times C_{P} \times\left(T_{\text {inj }}-T_{\text {pol }}\right)+\rho \times Q_{\text {avg }} \times H_{f}
$$

where $\rho, C_{p}$ and $H_{f}$ refer to the density, heat capacity and heat of fusion respectively; $T_{i n j}$ and $T_{p o l}$ refer to the injection temperature and initial temperature of the polymer; and $Q_{a v g}$ refers to the average flow rate of the polymer. The volume of each part is represented as $V_{\text {part }}$ and the volume of polymer used to obtain a volume of $V_{\text {part }}$ after including shrinkage in the polymer and buffer is given by $V_{\text {shot }}$. The volume of a shot is

$$
V_{\text {shot }}=V_{\text {part }} \times\left(1+\frac{\epsilon}{100}+\frac{\Delta}{100}\right)
$$

where $\epsilon$ and $\Delta$ represent the shrinkage in the polymer and buffer used in the process. Therefore, the energy $\left(E_{\text {melt }}\right)$ consumed in melting the polymer for a part is given by

$$
E_{\text {melt }}=\frac{P_{\text {melt }} \times V_{\text {shot }}}{Q}
$$

where $Q$ refers to the flow rate. The flow rate and the average flow rate are related as

$$
Q_{\text {avg }}=0.5 \times Q
$$

\subsubsection{Injection into mold}

In this process, the polymer dye in the liquid state is pressurized into the mold to form the required parts. The energy consumed in the injection process $\left(E_{i n j}\right)$ is given as

$$
E_{\text {inj }}=p_{\text {inj }} \times V_{\text {part }}
$$


where $p_{\text {inj }}$ is the injection pressure.

\subsubsection{Cooling of polymer}

The molten polymer in the mold is then cooled down to form the required part. The energy consumed $\left(E_{\text {cool }}\right)$ for the cooling process is given by

$$
E_{\text {cool }}=\frac{\rho \times V_{\text {part }} \times\left[C_{p} \times\left(T_{i n j}-T_{e j}\right)\right]}{C O P}
$$

where $T_{e j}$ refers to the ejection temperature, i.e., the temperature of the polymer when removed from the mold, and COP is the coefficient of performance of the cooling equipment. The total energy consumed in making a part is given by

$$
\begin{aligned}
E_{\text {part }}=\frac{1}{n} \times[ & \left(\frac{0.75 \times E_{\text {melt }}+E_{\text {inj }}}{\eta_{\text {inj }}}+\frac{E_{\text {reset }}}{\eta_{\text {reset }}}+\frac{E_{\text {cool }}}{\eta_{\text {cool }}}+\frac{0.25 \times E_{\text {melt }}}{\eta_{\text {heater }}}\right) \\
& \left.\times \frac{n \times(1+\epsilon+\Delta)}{\eta_{\text {machine }}}+P_{b} \times t_{\text {cycle }}\right]
\end{aligned}
$$

where $E_{\text {reset }}$ refers to the energy consumed in resetting the process, $P_{b}$ is the power required for basic energy consuming units when machine is in stand-by mode; $\eta_{\text {inj }}, \eta_{\text {reset }}, \eta_{\text {cool }}, \eta_{\text {heater }}$ and $\eta_{\text {machine }}$ refer to the efficiencies of injection, reset, cooling, heating and machine power respectively; and $t_{c y c l e}$ refers to the total cycle time. The reset energy is given by

$$
E_{\text {reset }}=0.25\left(E_{\text {inj }}+E_{\text {cool }}+E_{\text {melt }}\right)
$$

A Bayesian network can be constructed as shown in Fig. 7 to represent the causal dependencies between the variables described in Eq. (11) to (18) for the injection molding process. In the Bayesian network, the initial polymer temperature $\left(T_{p o l}\right)$, density $(\rho)$, heat capacity $\left(C_{p}\right)$ and 
shrinkage $(\epsilon)$ are assumed to have aleatory uncertainty while the following variables are assumed to have epistemic uncertainty - injection temperature, ejection temperature, mold temperature, injection pressure, flow rate, and measurement errors in, temperature, pressure, and flow.

Observations are assumed to be available on the flow rate, injection pressure, injection temperature, ejection temperature, mold temperature. Using the observations, the calibration of the epistemic variables is performed. A synthetic dataset of 100 observations is created using assumed true values of the parameters and by adding some measurement errors to the generated data. The measurement errors in temperature, and pressure are all assumed to have normal distributions with zero means and standard deviations of $3^{\circ} \mathrm{C}$, and $4 \mathrm{MPa}$ respectively.

The statistics of the parameters used to generate the dataset are given in Table 4. Polyethylene is assumed to be the polymer material used, and its assumed material property values are provided in Table 5. The next step is Bayesian model calibration for which the prior distributions of calibration parameters are given in Table 6 .

MCMC sampling (Metropolis-Hastings algorithm) is used to generate samples for the posterior distributions of calibration quantities. The posterior distributions are then used for estimation of the probability distribution of energy per part using Monte Carlo sampling and kernel density estimation as described in Section 3. The plots showing the prior and posterior distributions of the calibration parameters and energy per part are provided in Fig. 8.

To demonstrate the proposed dimension reduction methodology, global sensitivity analysis is carried out using both the prior and posterior distributions; the results are shown in Table 7. A first-order sensitivity threshold value of 0.001 is assumed. If the first-order sensitivity of a variable is less than the threshold value, the variable can be assumed to be deterministic as stated in Section IV. From the results of sensitivity analysis using prior distributions, it can be observed 
that none of the calibration parameters have sensitivity below the threshold value and therefore, all the variables are retained for calibration. After calibration, sensitivity analysis is again carried out using the posterior distributions. It can be observed that the posterior sensitivity indices are lower than the prior sensitivity indices for calibration parameters because the variance of the posterior distributions is much smaller than that of the prior distributions (Fig. 8). The posterior sensitivity indices of calibration parameters are still greater than threshold values; therefore the variation of these parameters is considered for further analysis.

The injection molding process as described above has three sub processes and the energy consumption of the overall process is the summation of energy consumption of each of the individual sub processes. The output of each sub process is an input to the overall energy consumption calculation. The same idea can be generalized for the calculation of energy consumption of a manufacturing network involving a number of individual processes. In other words, the Bayesian networks corresponding to the individual processes can be composed to form the Bayesian network of the entire production network, thus facilitating a modular and a hierarchical approach.

Apart from demonstrating the application of UQ and sensitivity analysis, the following observations are found from the results of the two numerical examples:

1. No significant difference can be seen between the prior and posterior of energy consumption in welding process; this implies that the dominant uncertainty sources in the welding example are aleatory (irreducible).

2. Significant uncertainty reduction can be seen for the energy consumption in the injection molding process due to significant updates in the epistemic variables, 
3. The posterior sensitivity indices of the epistemic variables in both injection molding and welding processes are quite small; therefore all the remaining uncertainty after calibration is mostly due to aleatory variables which cannot be further reduced.

The above observations bring out an important insight regarding resource allocation for uncertainty reduction. Only epistemic uncertainty is reducible; thus data collection is necessary for uncertainty reduction in the injection molding process, whereas data collection does not reduce the uncertainty in the welding process, which is mostly aleatory. The resource allocation decision could be made based on prior sensitivity indices (Tables 3 and 7), which also indicate that the energy prediction uncertainty in the welding process is dominated by aleatory sources, whereas the energy prediction uncertainty in the injection molding process is dominated by epistemic sources. Future work needs to consider heterogeneous information from different sources in different formats (e.g., numerical, text, image). Also in this study, the proposed methods were demonstrated using individual manufacturing processes.

\section{Conclusion}

This paper proposed a systematic approach for uncertainty quantification in the performance evaluation of manufacturing processes using Bayesian networks. The key features of the proposed methodology are construction of the Bayesian network, model calibration, forward uncertainty propagation and sensitivity analysis. The Bayesian network for the system (or process) can be constructed using first principles (physics-based models), using data (structure learning), or a hybrid approach using both domain knowledge and data. The next step is model calibration to estimate the unknown model parameters in the presence of observation data using sampling techniques such as MCMC. Using the posterior probability distributions, the 
probability distribution of the output variable (quantity of interest) can be obtained using Monte Carlo sampling.

Sensitivity analysis can be used for dimension reduction and uncertainty reduction decision making. The sensitivity results help to identify the parameters that have significant influence on the output uncertainty and also to separate the contributions of aleatory and epistemic uncertainty sources in the system to the uncertainty in the output of interest (energy consumption). The latter results can be used for efficient resource allocation for further data collection and/or model improvement. Also, variables that have sensitivity less than the specified threshold values can be assumed deterministic at their most probable values. The model calibration and forward uncertainty propagation procedures can then be carried out using the reduced set of variables.

The proposed methodologies are demonstrated with two manufacturing process examples welding and injection molding. As modeled in this paper, welding is a single step process whereas injection molding is a three-step process (heating, injection and cooling). Bayesian networks were constructed for UQ in both processes; the injection molding example with multiple steps demonstrates the ability to construct Bayesian networks for complicated manufacturing processes involving multiple individual processes in a hierarchical manner.

An important feature in the illustrative examples is that the Bayesian networks are constructed based on physics-based models. The mathematical equations are used to calculate the conditional probability relationships between parent and child nodes in the network. In many cases, such physics-based mathematical relationships may not be available, thus one may need to construct data-driven models. Two data analytics options are possible for constructing Bayesian networks in such situations. One could construct algebraic regression models from input-output data of the processes and use the regression equations in the same manner as in this paper. Another option is 
to directly learn the structure and nodal and conditional probabilities of the Bayesian network from the available data. A realistic implementation to manufacturing networks using a hybrid approach (i.e., physics-based models to infer some parts of the network and observational data to infer other parts) needs to be investigated in the future work. Note that the examples in this paper already fuse information from both models and data (for the nodes in the Bayesian network, but not the conditional probabilities) through the Bayesian calibration.

Future work needs to extend the proposed UQ methodology to production networks with multiple processes, some of which might interact with each other. The Bayesian networks in this paper were manually constructed based on available mathematical models for the two examples. Future work is needed to automate the generation of the Bayesian network for a manufacturing process using semantic descriptions, modeling libraries and leveraging existing knowledge and data in multiple organizations, in order to bring this technology closer to industrial use.

\section{Acknowledgment}

The research reported in this paper was supported by funds from the National Institute of

Standards and Technology under Smart Manufacturing Data Analytics Project (Cooperative Agreement No. 70NANB14H036). The support is gratefully acknowledged.

\section{References}

Bartram, G., Mahadevan, S., 2014. Integration of heterogeneous information in SHM models. Structural Control and Health Monitoring, 21(3), 403-422.

Bensi, M., Der Kiureghian, A., 2010. Seismic hazard modeling by Bayesian network and application to a high-speed rail system, Proceedings of International Symposium on Reliability Engineering and Risk Management, (Ed: J. Li), Tongji University Press, Shanghai, China. 
Brooks, S., 1998. Markov chain Monte Carlo method and its application, Journal of the Royal Statistical Society: Series D (the Statistician) 47.1: 69-100.

Castillo, E., Sarabia, J.M., Solares, C., Gomez, P., 1999. Uncertainty analysis in fault trees and Bayesian networks using FORM/SORM methods. Reliability Engineering and System Safety, 65(1): 29-40.

Dahll G., 2000. Combining disparate sources of information in the safety assessment of software-based systems, Nuclear Engineering and Design, 195: 307-319.

De Campos, L.M., Fernández-Luna, J.M., Huete, J.F., 2004. Bayesian networks and information retrieval: an introduction to the special issue, Information Processing \& Management, Elsevier, 40 (5): 727-733.

Deventer, R., Denzler, J., Niemann, H., 2000. Non-linear modeling of a production process by hybrid Bayesian networks, Proceedings, $14^{\text {th }}$ European Conference on Artificial Intelligence (Editor: W. Horn), Berlin, IOS Press.

Feng, SC., Senthilkumaran, K., Brown, C.U., Kulvatunyou, B., 2014. Energy metrics for product assembly equipment and processes, Journal of Cleaner Production 65: 142-151.

Friedman, N., Linial, M., Nachman, I., Pe’er, D., 2004. Using Bayesian Networks to Analyze Expression Data, Journal of Computational Biology, (3/4): 601-620.

Heckerman, D., Geiger, D., Chickering, D. M., 1995. Learning Bayesian networks: The combination of knowledge and statistical data, Machine learning, 20(3), 197-243.

Jensen, F. V., 1996. An Introduction to Bayesian Networks,UCL Press, London.

Jiang, X., Cooper, G.F., 2010. A Bayesian Spatio-Temporal Method for Disease Outbreak Detection, Journal of American Medical Informatics Association 17 (4): 462-71. 
Jiang, X., Neapolitan, R.E., Barmada, M.M., Visweswaran, S., 2011. Learning Genetic Epitasis using Bayesian Network Scoring Criteria, BMC Bioinformatics 12: 89.

Kohavi, R., John, G. H., 1997. Wrappers for feature subset selection, Artificial intelligence, 97(1), 273-324.

Liang, B., Mahadevan, S., 2011. Error and uncertainty quantification and sensitivity analysis in mechanics computational models, International Journal for Uncertainty Quantification, Vol 1(2), pp.147-161.

Ling, Y., Mahadevan, S., 2013. Bayesian Networks for Comprehensive Uncertainty Quantification in Multi-Scale Multi-Physics Analysis, Proceedings, 9th International Conference on Structural Safety and Reliability (ICOSSAR), New York.

Madan, J., Mani. M., Lyons, K.W., 2013. Characterizing Energy Consumption of the Injection Molding Process, International Manufacturing Science and Engineering Conference collocated with the 41st North American Manufacturing Research Conference, American Society of Mechanical Engineers.

Mahadevan, S., Zhang, R., Smith, N., 2001. Bayesian Networks for System Reliability Reassessment, Structural Safety, Vol. 23 (3), pp. 231-251.

McNaught, K., Chan, A., 2011. Bayesian networks in manufacturing, Journal of Manufacturing Technology Management, Vol. 22, No. 6, pp. 734-747.

Pehlken, A., Decker, A., Kottowski, C., Kirchner, A., Thoben, K. D., 2015. Energy efficiency in processing of natural raw materials under consideration of uncertainties. Journal of Cleaner Production, Vol. 106, pp. 351-363

Rangavajhala, S., Sura, V. S., Hombal, V. K., Mahadevan, S., 2011. Discretization Error Estimation in Multidisciplinary Simulations, AIAA Journal, 49(12), 2673-2683. 
Rao, S., Strojwas, A., Lehocsky, J., Schervish, M., 1995. Monitoring multi-stage sequential manufacturing processes: a Bayesian approach, Proceedings, IEEE /UCS/SEMI International Symposium on Semiconductor Manufacturing, Austin, TX.

Rebba, R., Mahadevan, S., Huang, S., 2006. Validation and error estimation of computational models, Reliability Engineering and System Safety, 91(10), 1390-1397.

Reza, B., Sadiq, R., Hewage, K., 2013. A fuzzy-based approach for characterization of uncertainties in emergy synthesis: an example of paved road system. Journal of Cleaner Production, 59, 99-110.

Richards, S. A., 1997. Completed Richardson Extrapolation in Space and Time, Communications in Numerical Methods in Engineering, 12, 573-582.

Rodrigues, M.A., Liu, Y., Bottaci, L., Rigas, D.I., 2000. Learning and Diagnosis in manufacturing processes through an executable Bayesian network," Proceedings, $13^{\text {th }}$ International Conference on Industrial Engineering Applications of Artificial Intelligence and Expert Systems, pp. 390-395, Springer-Verlag, New York.

Saeys, Y., Inza, I., Larranaga, P., 2007. A review of feature selection techniques in bioinformatics, Bioinformatics, 23(19), 2507-2517.

Sankararaman, S., Mahadevan, S., 2011a. Likelihood-based representation of epistemic uncertainty due to sparse point data and interval data, Reliability Engineering and System Safety, Vol. 96, No. 7, pp. 814-824.

Sankararaman, S., Ling, Y., Mahadevan, S., 2011b. Uncertainty quantification and model validation of fatigue crack growth prediction, Engineering Fracture Mechanics, 78(7), 14871504. 
Sankararaman, S, 2012. Uncertainty quantification and integration in engineering systems, $\mathrm{PhD}$ dissertation, Dept., Civil Eng., Vanderbilt Univ, Nashville, TN.

Sankararaman, S., Mahadevan, S., 2013a. Separating the contributions of variability and parameter uncertainty in probability distributions. Reliability Engineering and System Safety, 187-199.

Sankararaman, S., McLemore, K., Mahadevan, S., 2013b. Test Resource Allocation in Hierarchical Systems using Bayesian Networks, AIAA Journal, Vol. 51, No. 3, pp. 537-550.

Scutari, M., 2010. Learning Bayesian Networks with the bnlearn R Package. Journal of Statistical Software, 35(3), 1-22.

Sonnemann, G. W., Schuhmacher, M., Castells, F., 2003. Uncertainty assessment by a Monte Carlo simulation in a life cycle inventory of electricity produced by a waste incinerator. Journal of Cleaner Production, 11(3), 279-292.

Toni, T., Welch, D., Strelkowa, N., Ipsen, A., Stumpf, M.P.H., 2009. Approximate Bayesian computation scheme for parameter inference and model selection in dynamical systems, Journal of the Royal Society Interface 6.31: 187-202.

Urbina, A., Mahadevan, S., Paez, T.L., 2012. A Bayes Network Approach to Uncertainty Quantification in Hierarchically Developed Computational Models, International Journal for Uncertainty Quantification. Vol. 2, No. 2, pp. 173-193.

Weber, P., Jouffe, L., 2006. Complex system reliability modeling with dynamic object-oriented Bayesian networks (DOOBN), Reliability Engineering and System Safety, Vol. 01, pp. 149162. 
Yu, L., Heng, Z., 2006. Measuring Agility of Enterprise using Analytic Hierarchy Process and Bayesian Belief Networks, Proceedings, International Conference on Management Science and Engineering, Lille, France, pp. 551-556. 
Figure 1

Figure 2

Figure 3

Figure 4

Figure 5

Figure 5

Figure 6

Figure 7

Figure 7

Figure 8
A simple Bayesian network with three variables Uncertainty Quantification Methodology

Dimension reduction using global sensitivity analysis

Weld zone parameters (Feng et al; 2014).

Bayesian network for welding process

Bayesian network for welding process

Prior and Posteriors of Welding parameters (i) Mean of $l$ (ii) Standard deviation of $l$ (iii) Mean of $h$ (iv) Standard deviation of $h$ (v) Mean of $e$ (vi) Standard deviation of $e$ (vii) Theoretical minimum energy Bayesian network for injection molding process Bayesian network for injection molding process

Prior and Posterior probability distributions of Injection Molding parameters (i) Injection temperature (ii) Ejection temperature (iii) Mold temperature (iv) Injection pressure (v) Flow rate (vi) Energy per part

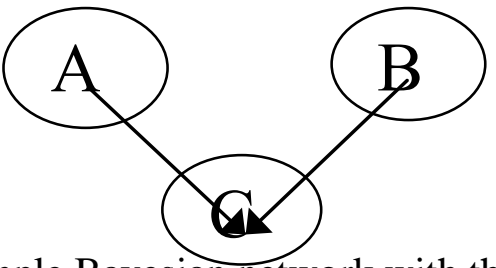

Fig. 1. A simple Bayesian network with three variables 


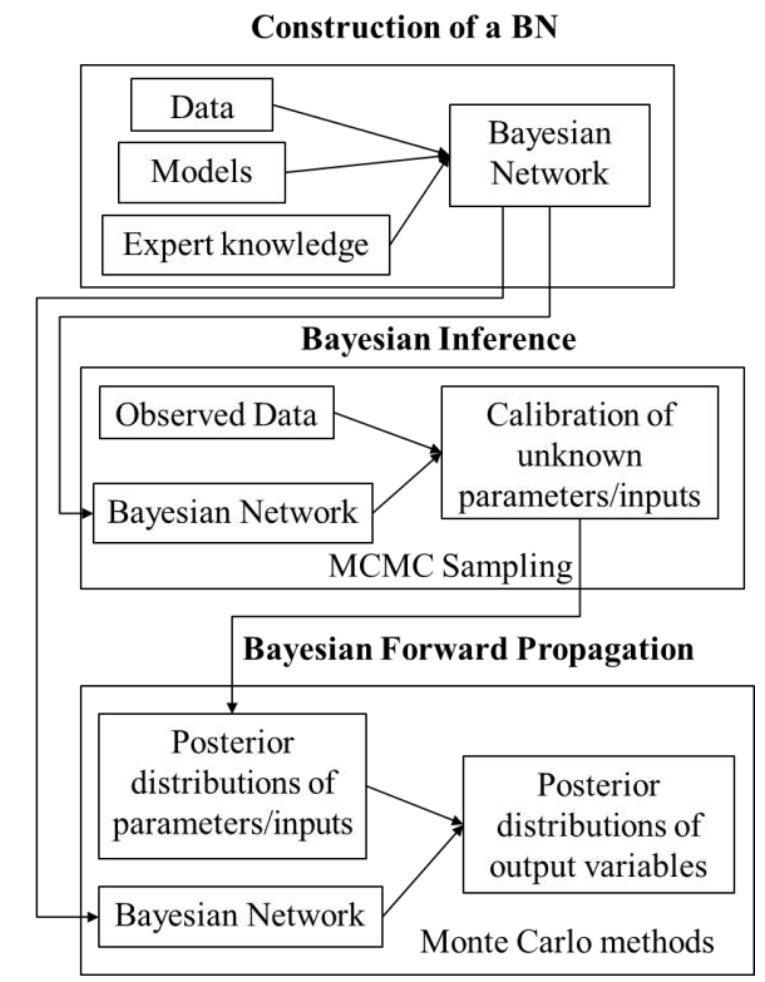

Fig. 2. Uncertainty Quantification methodology

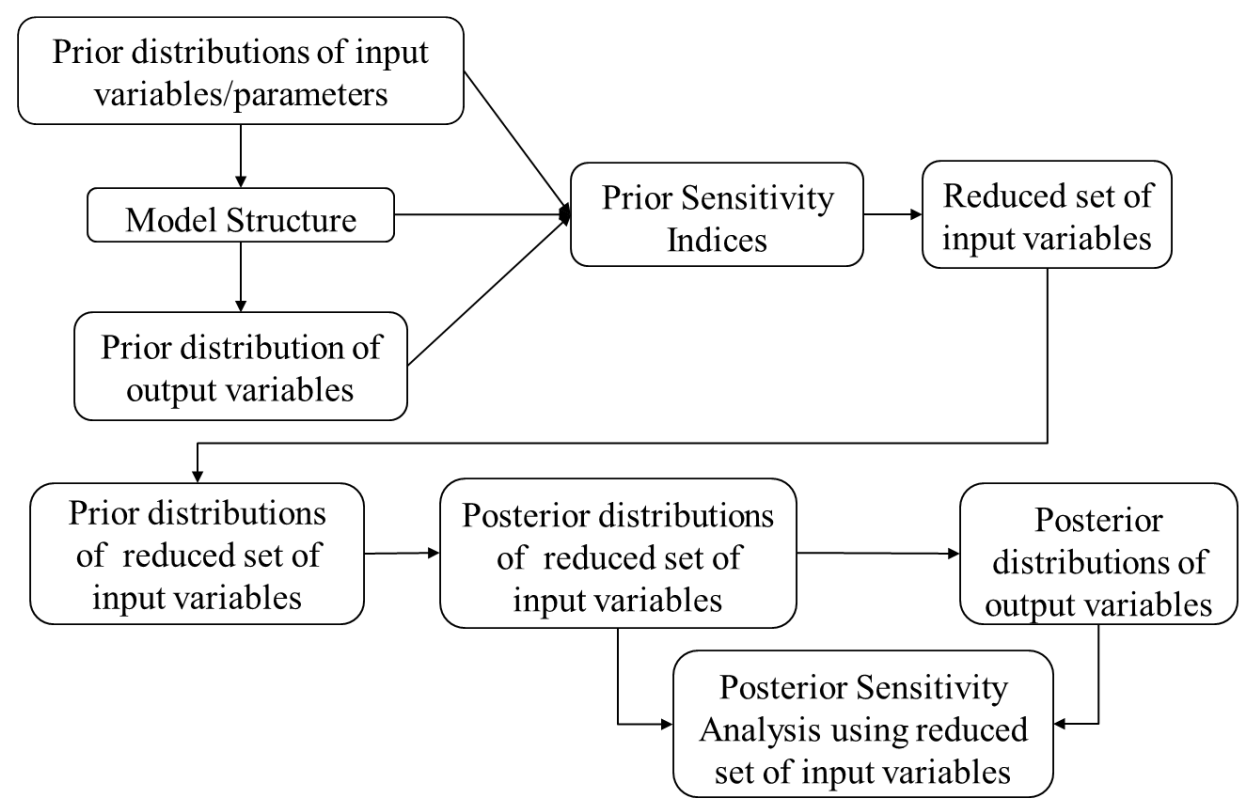

Fig. 3. Dimension reduction using global sensitivity analysis 


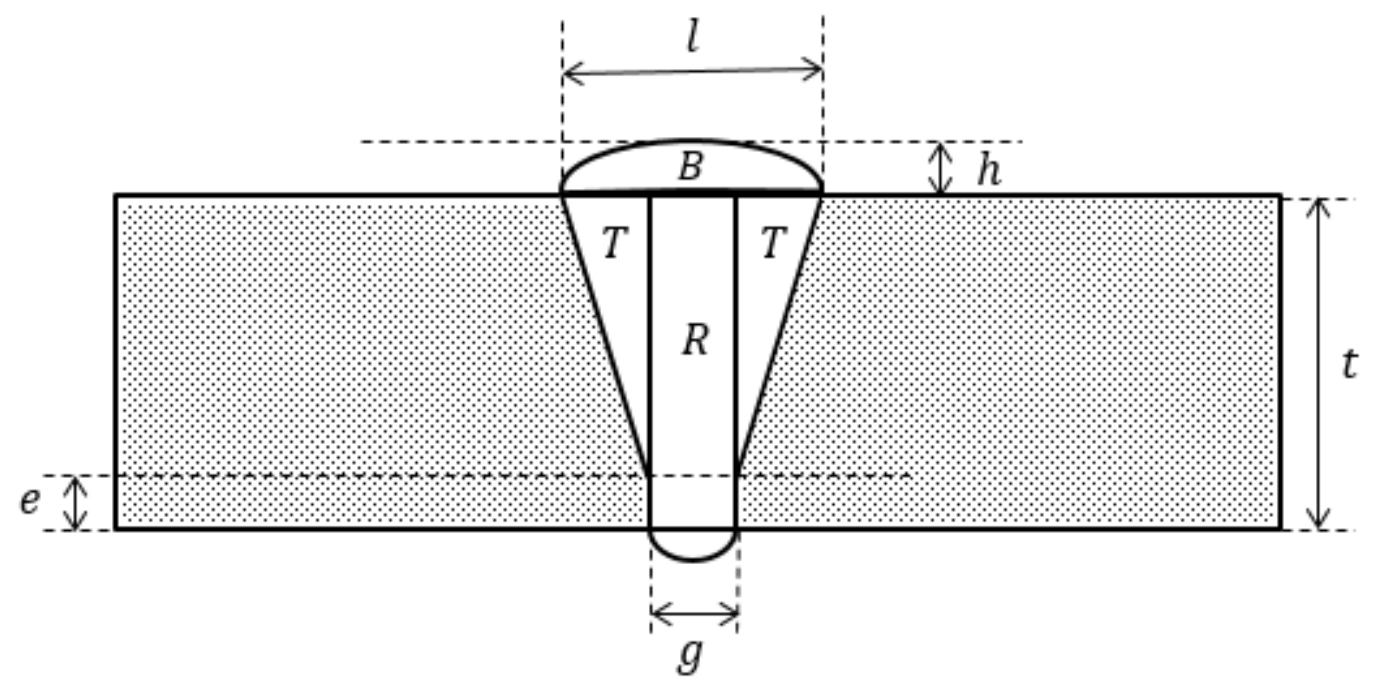

Fig. 4. Weld zone parameters (Feng et al; 2014). 


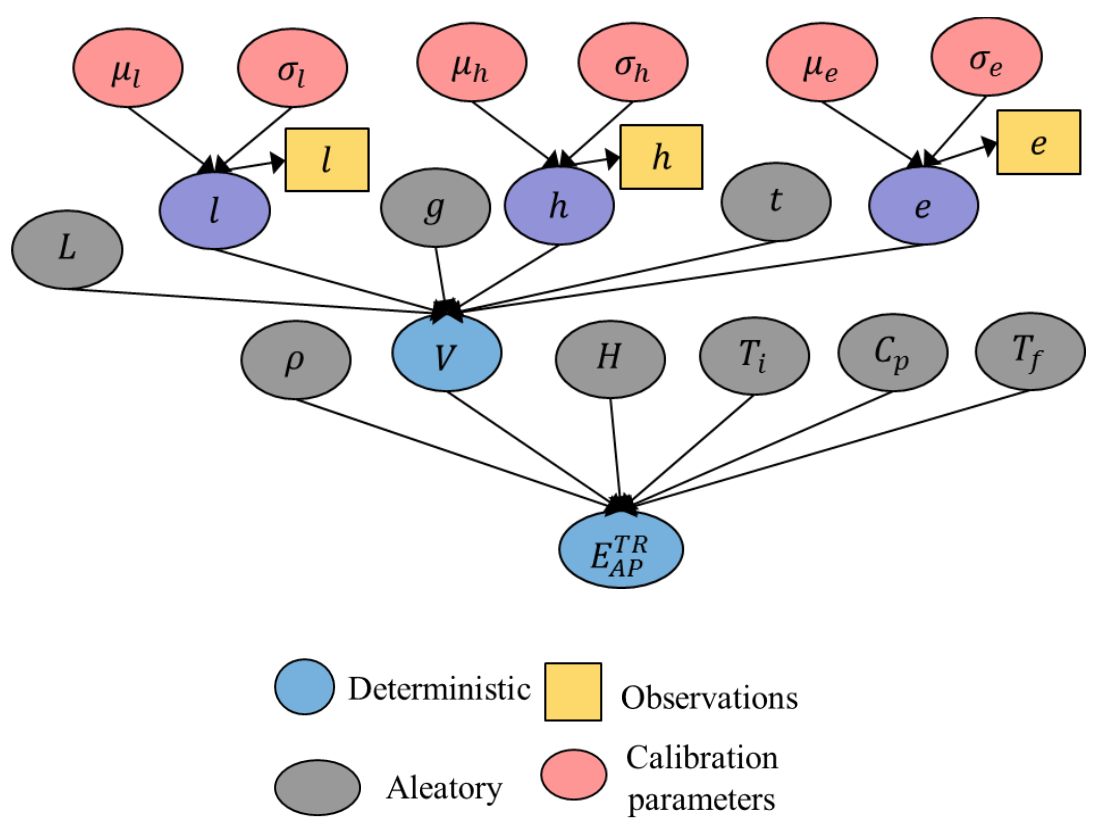

Fig. 5. Bayesian network for the welding process

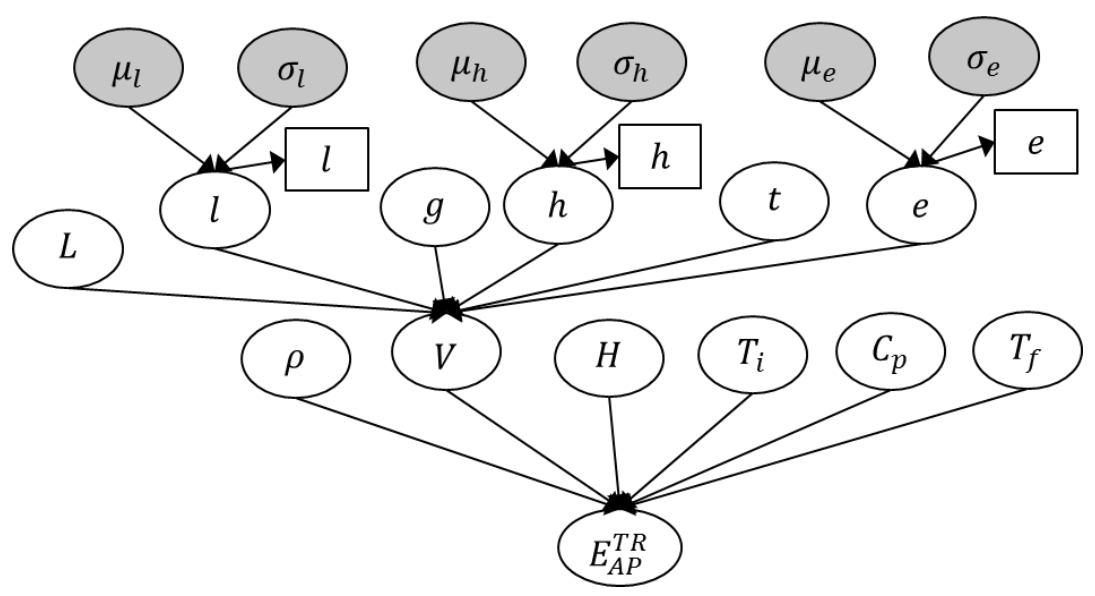




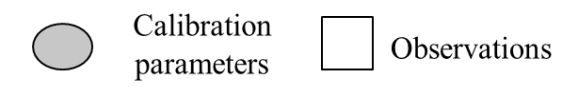

Fig. 5. Bayesian network for the welding process

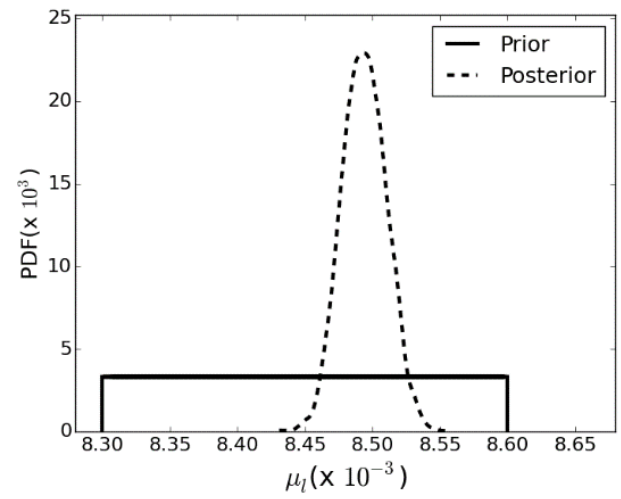

(i)

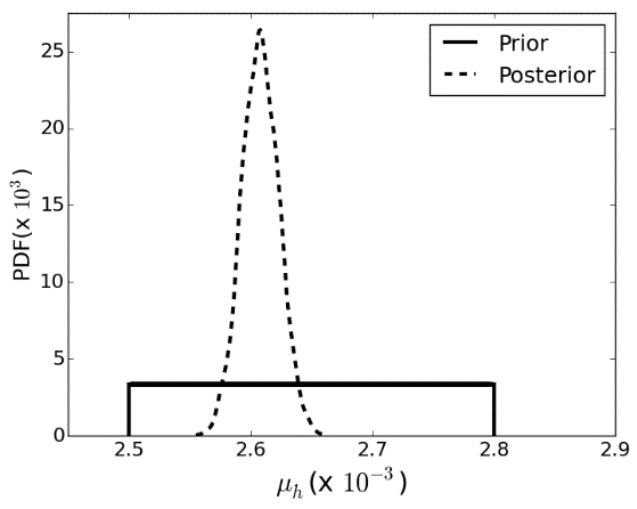

(iii)

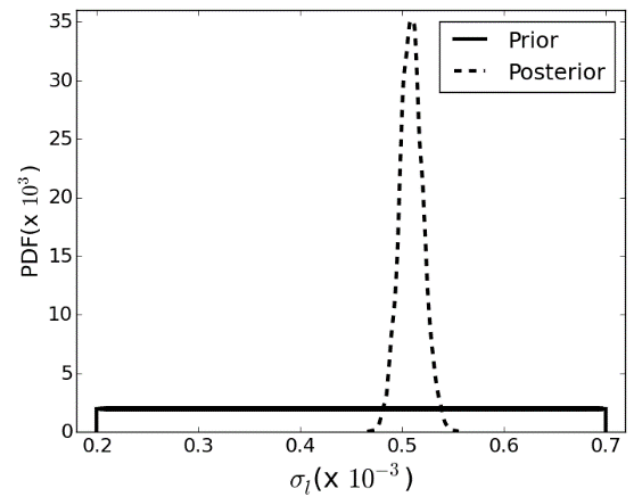

(ii)

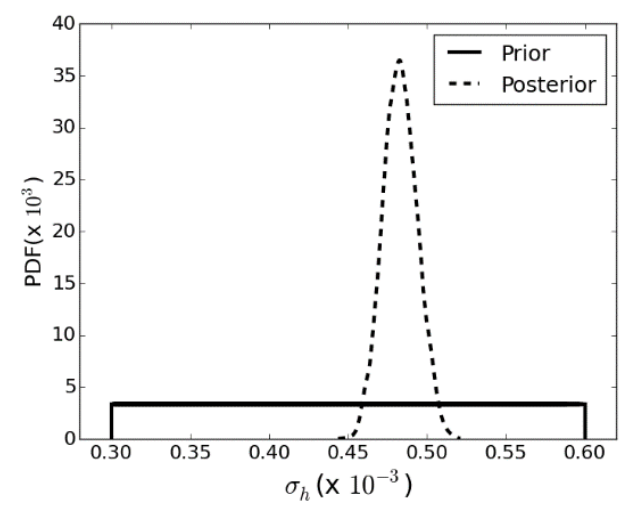

(iv) 


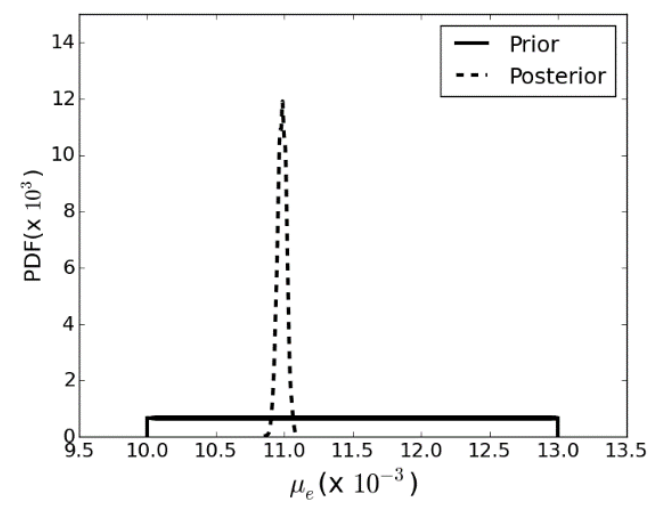

(v)

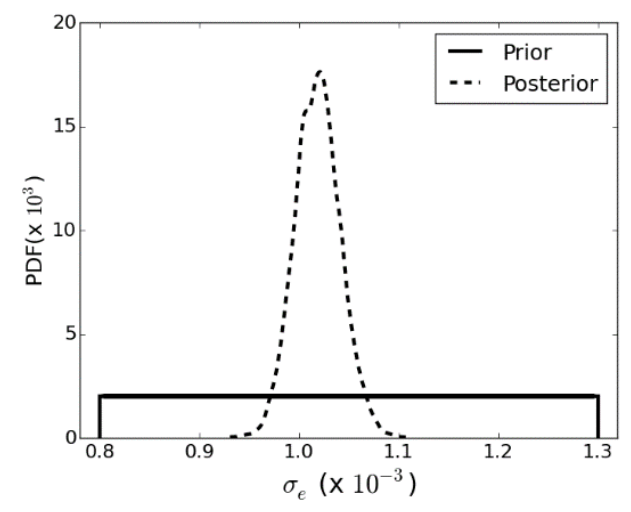

(vi)

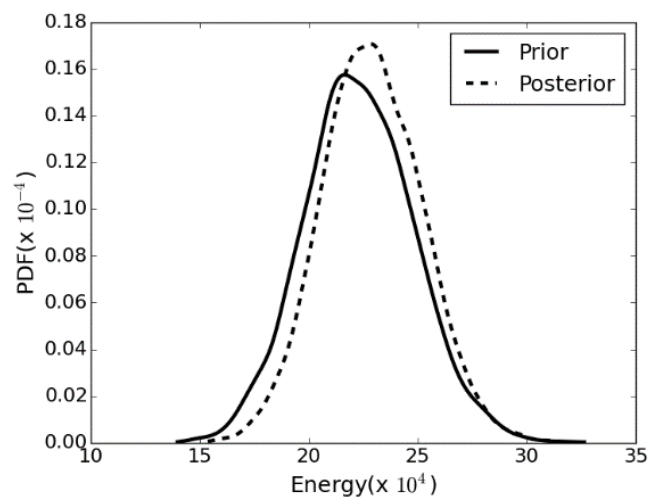

(vii)

Fig. 6. Prior and posteriors of welding parameters: (i) Mean of $l$ (ii) Standard deviation of $l$ (iii) Mean of $h$ (iv) Standard deviation of $h$ (v) Mean of $e$ (vi) Standard deviation of $e$ (vii) Theoretical minimum energy 


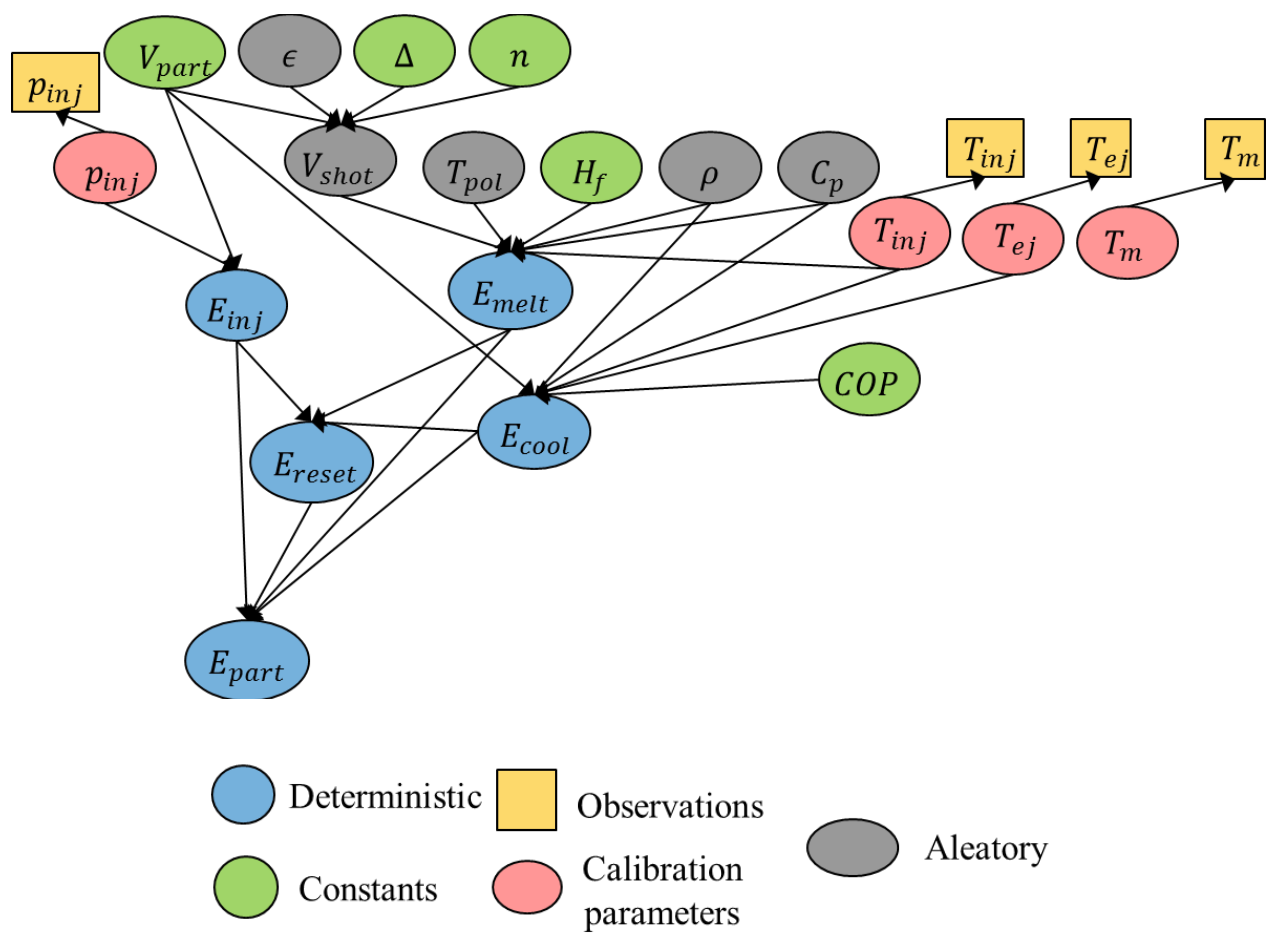

Fig. 7. Bayesian network for the injection molding process 


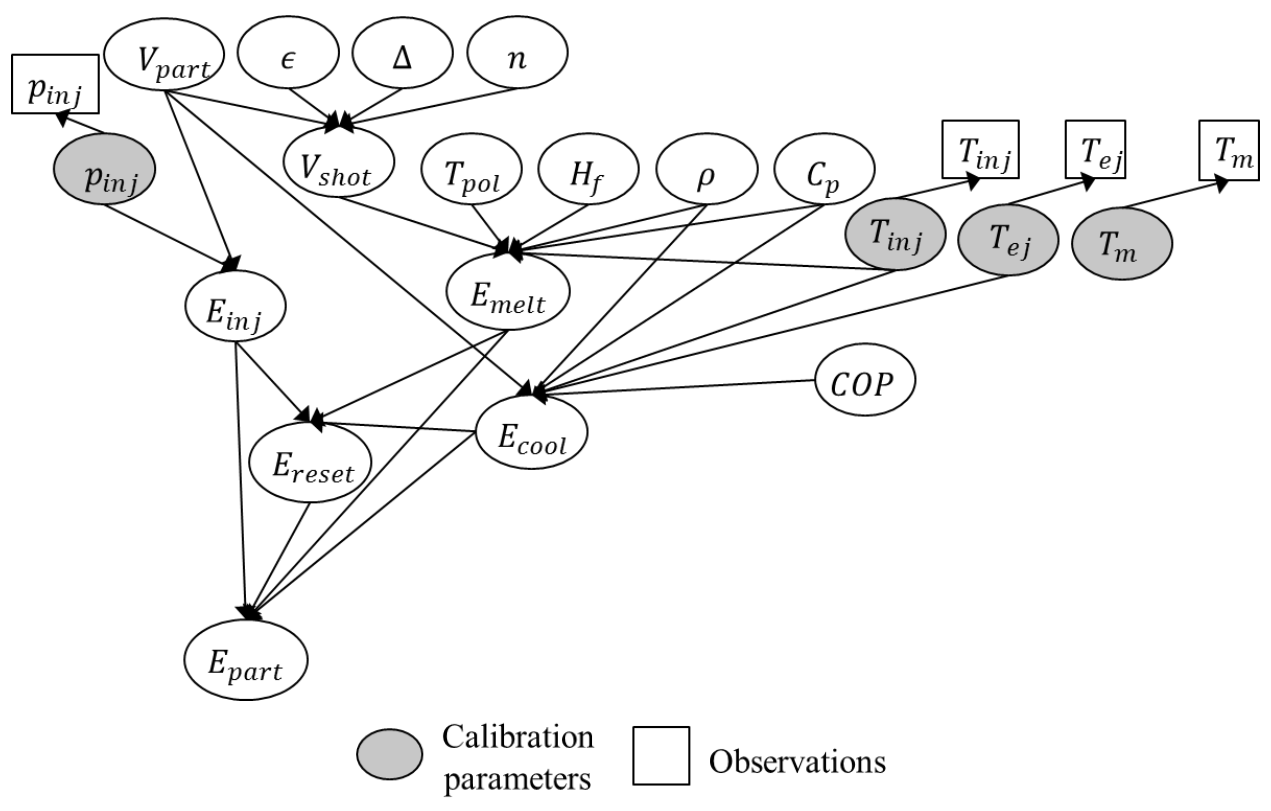

Fig. 7. Bayesian network for the injection molding process

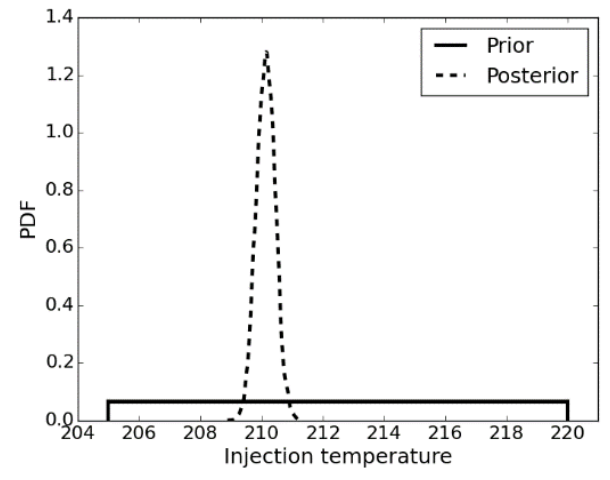

(i)

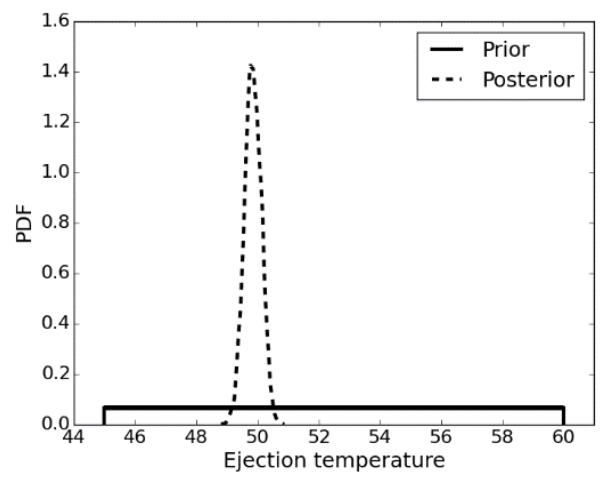

(ii) 


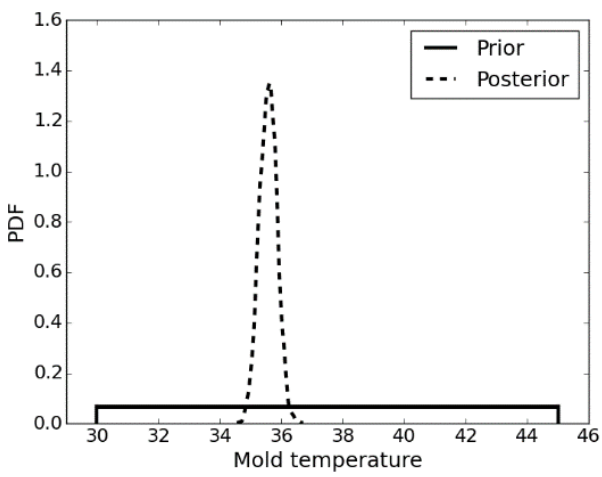

(iii)

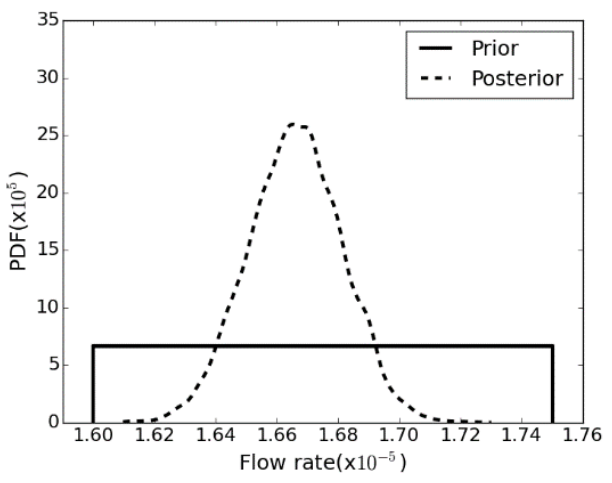

(v)

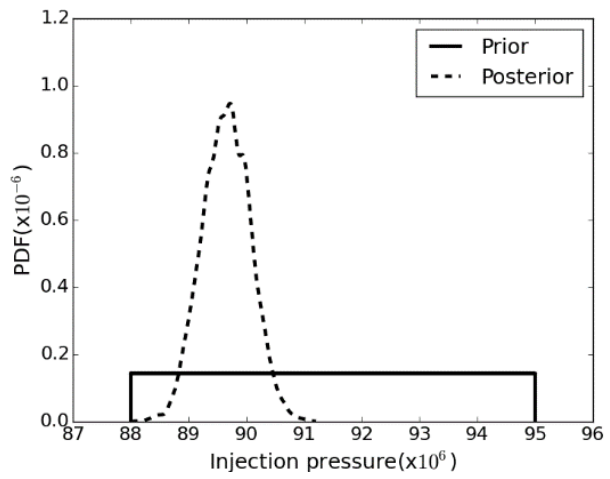

(iv)

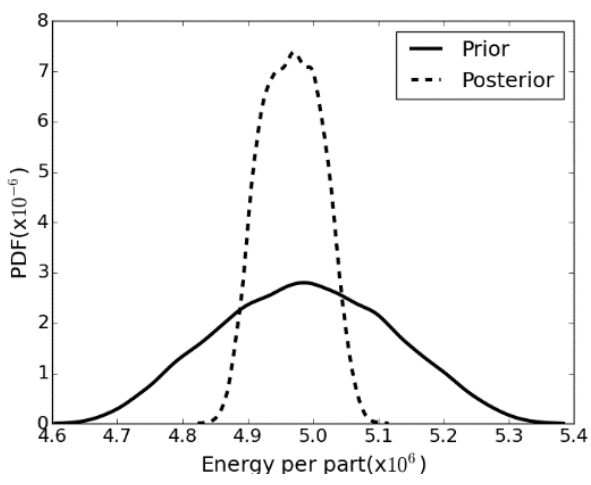

(vi)

Fig. 8. Priors and posteriors of injection molding parameters: (i) Injection temperature (ii) Ejection temperature (iii) Mold temperature (iv) Injection pressure (v) Flow rate (vi) Energy per part 
Table $1 \quad$ Parameters for synthetic dataset generation: welding process

Table 2 Prior distributions of calibration parameters: welding process

Table 3 Prior and posterior sensitivity indices of parameters: welding process

Table 4 Parameters for synthetic dataset generation: injection molding process

Table $5 \quad$ Material properties of polyethylene polymer

Table 6 Prior distributions of calibration parameters: injection molding process

Table 7 Prior and posterior sensitivity indices of parameters: injection molding process 
Table 1. Parameters for synthetic dataset generation: welding process

\begin{tabular}{cc}
\hline Parameter & Statistics \\
\hline Initial temperature $\left(T_{i}, K\right)$ & $N(303,0.3)$ \\
Final temperature $\left(T_{f}, K\right)$ & $N(1628,10)$ \\
Heat capacity $\left(C_{p}, J /(k g K)\right)$ & $N(500,5)$ \\
Density $\left(\rho, k g / m^{3}\right)$ & $N(8238,10)$ \\
Latent Heat $(H, k J / k g)$ & $N(270,3)$ \\
Weld parameters $(m m)$ & \\
$l$ & $N(8.5,0.5)$ \\
$h$ & $N(2.6,0.5)$ \\
$g$ & $N(2,0.1)$ \\
$t$ & $N(15,0.5)$ \\
$e$ & $N(11,1)$ \\
Length of weld $(L, m m)$ & $N(500,10)$ \\
\hline
\end{tabular}


Table 2. Prior distributions of calibration parameters: Welding process

\begin{tabular}{ccc}
\hline Parameter & Statistics \\
\hline$l$ & $\mu_{l}$ & $U(8.3,8.6)$ \\
& $\sigma_{l}$ & $U(0.2,0.7)$ \\
\hline \multirow{2}{*}{$h$} & $\mu_{h}$ & $U(2.5,2.8)$ \\
& $\sigma_{h}$ & $U(0.3,0.6)$ \\
\hline & $\mu_{e}$ & $U(10,13)$ \\
$e$ & $\sigma_{e}$ & $U(0.8,1.3)$ \\
\hline
\end{tabular}


Table 3. Prior and Posterior sensitivity indices of parameters: welding process

\begin{tabular}{|c|c|c|c|c|c|c|}
\hline \multicolumn{2}{|r|}{ Parameter } & \multirow{2}{*}{$\begin{array}{c}\text { Type of } \\
\text { uncertainty } \\
\text { Aleatory }\end{array}$} & \multirow{2}{*}{$\begin{array}{c}\begin{array}{c}\text { Prior - } \\
\text { Individual } \\
\text { effect }\end{array} \\
0.0745\end{array}$} & \multirow{2}{*}{$\begin{array}{c}\text { Prior - total } \\
\text { effect }\end{array}$} & \multirow{2}{*}{$\begin{array}{c}\text { Posterior - } \\
\begin{array}{c}\text { Individual } \\
\text { effect }\end{array} \\
0.1193\end{array}$} & \multirow{2}{*}{$\begin{array}{c}\text { Posterior - } \\
\text { total effect } \\
0.1226\end{array}$} \\
\hline & $u_{l}$ & & & & & \\
\hline \multirow[t]{3}{*}{$l$} & $\mu_{l}$ & Epistemic & 0.0026 & 0.0027 & 0.00012 & 0.00013 \\
\hline & $\sigma_{l}$ & Epistemic & $6.44 \times 10^{-7}$ & $7.75 \times 10^{-3}$ & $1.062 \times 10^{-7}$ & $6.005 \times 10^{-5}$ \\
\hline & $u_{h}$ & Aleatory & 0.2147 & 0.2238 & 0.2995 & 0.3001 \\
\hline \multirow[t]{3}{*}{$h$} & $\mu_{h}$ & Epistemic & 0.0073 & 0.0074 & 0.00025 & 0.000247 \\
\hline & $\sigma_{h}$ & Epistemic & $7.056 \times 10^{-8}$ & 0.00826 & $7.208 \times 10^{-10}$ & 0.000135 \\
\hline & $u_{e}$ & Aleatory & 0.3153 & 0.3227 & 0.2967 & 0.2989 \\
\hline \multirow[t]{2}{*}{$e$} & $\mu_{e}$ & Epistemic & 0.2019 & 0.2032 & 0.00034 & 0.00034 \\
\hline & $\sigma_{e}$ & Epistemic & $1.225 \times 10^{-6}$ & 0.00569 & $2.991 \times 10^{-9}$ & 0.000147 \\
\hline & $g$ & Aleatory & 0.044 & 0.0442 & 0.0493 & 0.0494 \\
\hline & $t$ & Aleatory & 0.1651 & 0.1655 & 0.2069 & 0.2075 \\
\hline & $\rho$ & Aleatory & 0.000124 & 0.000125 & 0.000153 & 0.000154 \\
\hline & $C_{p}$ & Aleatory & 0.00435 & 0.0044 & 0.00549 & 0.00554 \\
\hline & $H$ & Aleatory & 0.00091 & 0.00093 & 0.00107 & 0.00108 \\
\hline & $T_{i}$ & Aleatory & $2.393 \times 10^{-6}$ & $2.421 \times 10^{-6}$ & $2.054 \times 10^{-6}$ & $2.564 \times 10^{-6}$ \\
\hline & $T_{f}$ & Aleatory & 0.00225 & 0.00228 & 0.00313 & 0.00316 \\
\hline & $L$ & Aleatory & 0.0347 & 0.0351 & 0.04097 & 0.0413 \\
\hline
\end{tabular}


Table 4. Parameters for synthetic dataset generation: injection molding process

\begin{tabular}{cc}
\hline Parameter & Statistics \\
\hline Injection temperature $\left(T_{\text {in } j},{ }^{\circ} C\right)$ & 210 \\
Ejection temperature $\left(T_{e j},{ }^{\circ} C\right)$ & 35 \\
Mold temperature $\left(T_{m},{ }^{\circ} C\right)$ & 50 \\
Injection pressure $\left(p_{\text {inj }}, M P a\right)$ & 90 \\
Flow rate $\left(Q, m^{3} / s\right)$ & $1.67 \times 10^{-5}$ \\
Coefficient of performance $(C O P)$ & 0.7 \\
Energy coefficients $(\eta)$ & 0.7 \\
Number of cavities $(n)$ & 1 \\
Fraction $(\Delta)$ & 0.015 \\
Thickness $\left(h_{\text {max }}, m\right)$ & 0.0125 \\
Volume of part $\left(V_{\text {part }}, m^{3}\right)$ & 0.002048 \\
\hline
\end{tabular}


Table 5. Material properties of polyethylene polymer

\begin{tabular}{cc}
\hline Parameter & Value \\
\hline Density $\left(\rho, \mathrm{kg} / \mathrm{m}^{3}\right)$ & $U(950,990)$ \\
Heat capacity $\left(C_{p}, J /(\mathrm{kg} /)\right)$ & $U(2250,2260)$ \\
Thermal diffusivity $\left(\gamma, \mathrm{m}^{2} / \mathrm{s}\right)$ & $2.27 \times 10^{-7}$ \\
Shrinkage $(\epsilon)$ & $U(0.018,0.021)$ \\
Heat of fusion $\left(H_{f}, \mathrm{~kJ} / \mathrm{kg}\right)$ & 240 \\
\hline
\end{tabular}


Table 6. Prior distributions of calibration parameters: injection molding process

\begin{tabular}{cc}
\hline Parameter & Statistics \\
\hline Injection temperature $\left(T_{i n j},{ }^{\circ} \mathrm{C}\right)$ & $U(205,220)$ \\
Ejection temperature $\left(T_{e j},{ }^{\circ} \mathrm{C}\right)$ & $U(45,60)$ \\
Mold temperature $\left(T_{m},{ }^{\circ} \mathrm{C}\right)$ & $U(30,45)$ \\
Injection pressure $\left(p_{i n j}, M P a\right)$ & $U(88,95)$ \\
Flow rate $\left(Q, \mathrm{~m}^{3} / \mathrm{s}\right)\left(\times 10^{-5}\right)$ & $U(1.6,1.75)$ \\
\hline
\end{tabular}


Table 7. Prior and Posterior sensitivity indices of parameters: injection molding process

\begin{tabular}{cccccc}
\hline Variable & $\begin{array}{c}\text { Type of } \\
\text { Uncertainty }\end{array}$ & $\begin{array}{c}\text { Prior }- \\
\text { Individual effect }\end{array}$ & $\begin{array}{c}\text { Prior - } \\
\text { Total effect }\end{array}$ & $\begin{array}{c}\text { Posterior }- \\
\text { Individual effect }\end{array}$ & $\begin{array}{c}\text { Posterior - } \\
\text { Total effect }\end{array}$ \\
\hline$T_{i n j}$ & Epistemic & 0.4857 & 0.5829 & 0.0083 & 0.0125 \\
$T_{p o l}$ & Aleatory & 0.0161 & 0.0363 & 0.0686 & 0.0961 \\
\cline { 2 - 6 }$T_{e j}$ & Epistemic & 0.2412 & 0.3617 & 0.0063 & 0.0126 \\
$\rho$ & Aleatory & 0.1226 & 0.1264 & 0.7997 & 0.818 \\
\cline { 2 - 6 }$P_{i n j}$ & Epistemic & 0.0028 & 0.0078 & 0.0018 & 0.0019 \\
\cline { 2 - 6 }$\epsilon$ & Aleatory & 0.0015 & 0.0035 & 0.0107 & 0.0418 \\
\cline { 2 - 6 }$C_{p}$ & Aleatory & 0.0134 & 0.0137 & 0.0414 & 0.0798 \\
\hline
\end{tabular}

